\title{
Increased Cortical Representation of the Fingers of the Left Hand in String Players
}

\author{
Thomas Elbert, Christo Pantev, Christian Wienbruch, \\ Brigitte Rockstroh, Edward Taub
}

Magnetic source imaging revealed that the cortical representation of the digits of the left hand of string players was larger than that in controls. The effect was smallest for the left thumb, and no such differences were observed for the representations of the right hand digits. The amount of cortical reorganization in the representation of the fingering digits was correlated with the age at which the person had begun to play. These results suggest that the representation of different parts of the body in the primary somatosensory cortex of humans depends on use and changes to conform to the current needs and experiences of the individual.

Evidence has accumulated over the past two decades that indicarcs that alterations in afferent input can induce plastic reorganizational changes within the adult mammalian central nervous system (1). Changes in the relation between peripheral sensory fields and their central representations have been observed for the somatosensory (2), visual $(1,3,4)$, and auditory systems (5), and comparable changes also have been found for motor systems (6). In many of these experiments, the removal of afferent input from a cortical region resulted in an "invasion" by a neighboring area whose innervation remained intact. For example, the cortical region representing a digit before amputation in owl monkeys could be activated after amputation by tactile stimulation of an intact adjacent finger (7). The changes noted were of the order of a few millimeters. More extensive plastic changes have recently been observed after the abolition of input from larger portions of the body-for example, with somatosensory deafferentation of an entire forelimb in macaque monkeys (8) and upper extremity amputation in humans $(9-11)$.

In addition, it has heen shown in studies with owl monkeys that a prolonged increase of tactile stimulation to the distal pad of one or two phalanges results in a greatly increased cortical representation specific to that portion of the fingers $(12,13)$. Evidence has also been reported that suggests an increased corrical representation of the index finger used in reading by blind Braille readers ( 14$)$

Violinists and other string plavers provide a good model for the study of the effects of differential afferent input to the two sides of the brain in humans. During their practice or performance, the second to the fifth digits (122 to D5) of the left hand are continuously engaged in fingering the strings, a task that involves considerable manual dexterity and enhanced sensory stimulation. At the same time, the thumb grasps the neck of the instrument and, al- though not as active as the fingers, engages in relatively frequent small shifts of position and pressure. The right hand, which manipulates the bow, participates in a task involving much less individual finger movement and fluctuation in tacrile and pressure input. Here, we present data from magnetic source imnging that indicates that the cerebral cortices of string players are different from the cortices of controls in that the representation of the digits of the left hand is substantially enlarged in the cortices of string players

Nine musicians (six violinists, two cel lists, and one guitarist) who had played their instruments for a mean period of 11.7 years (range, 7 to 17 years) served as subjects for our study. Six nonmusicians served as controls (15). The mean age for bork groups was $24 \pm 3$ years. Before our inv tigation, the musicians kept a diary for 1 week, recording the amount of time practiced per day (mean $9.8 \pm 8.4$ hours por week), and had estimated the amount of time spent practicing during the previous month and year $(10.8 \pm 8.8$ hours per week).

During the experimental session, somatosensory stimulation was delivered to the first digit and, in separate runs, to the fifth digit of either hand. Stimulation consisted of light superficial pressure applied by means of a pneumatic stimulator with the use of standard, nonpainful stimulation intensity $(9,16,17)$. The data (Fig. 1) indicate that the center of cortical responsivity for tactile stimulation of the digits of the left hand was shifted in musicians as compared to that in controls, while at the same time the strength of response increased. The topouraphic shift was toward the mir sagittal plane, which, along the surface the postcentral gyrus, is toward the region
A

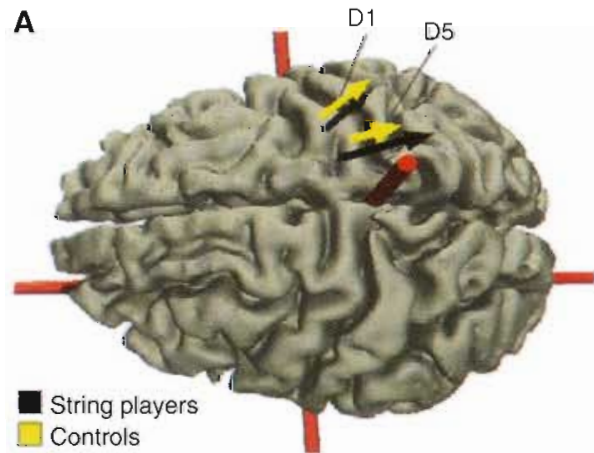

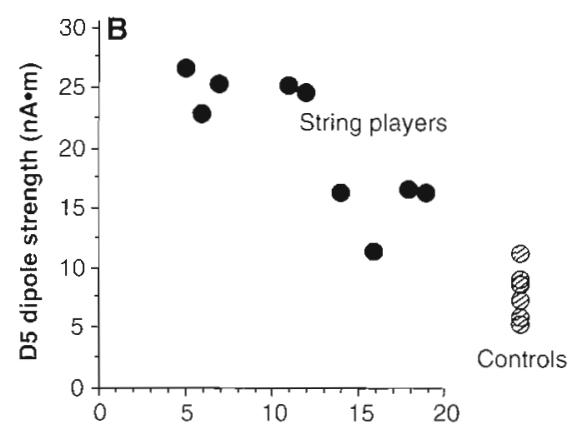

Age at inception of musical practice

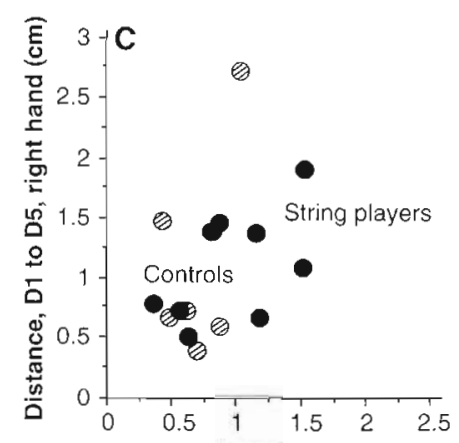

Distance, D1 to D5, left hand $(\mathrm{cm})$
Fig. 1. (A) Equivalent current dipoles elicited by stimulation of the thumb (D1) and fifth finger (D5) of the left hand are superimposed onto an MRI (magnetic resonance imaging) reconstruction of the cerebral cortex of a control, who was selected to provide anatomical landmarks for the interpretation of the MEG-based localization. The arrows represent the location and orientation of the ECD vector for each of the two digits averaged across musicians (black) and controls (yellow). The length of the arrows represents the mean magnitude of the dipole moment for the two digits in each group. The average locations of D5 and D1 are shifted medially for the string players compared to controls; the shift is larger for D5 than for D1. The dipole moment is also larger for the musicians' D5, as indicated by the greater magnitude of the black arrow. (B) The magnitude of the dipole moment as a function of the age of inception of musical practice; string players are indicated by filled circles, control subjects by hatched circles. Note the larger dipole moment for individuals beginning musical practice before the age of 12 . (C) Scatterplot of the Euclidean distances (in centimeters) between the cortical representations of D1 and D5. This distance for the musicians' left hands was greater than that in controls, but this difference is not statistically significant. 
of the cortex that represents the palm of the hand (18). For D5 (little finger) of the left hand, the shift was $0.7 \mathrm{~cm}(t=3.6, P<$ 0.01 , degrees of freedom $=13$ ); for $D 1$ (thumb), the shift was $0.5 \mathrm{~cm}(t=3.3, P<$ 0.01 ). This shift of D5 was significantly greater than the shift of D1.

Correspondingly, the analysis of variance (ANOVA), which included data from both hemispheres (18), showed an interaction of group (a factor in ANOVA) and digit $[F(1,13)=4.78, P<0.05]$. This effect was dependent on the side on which stimulation was given: There were no significant shifts for the digits of the right hand of the musicians compared to those of the controls. The dipole moment, which is presumed to be an index of total neuronal rctivity, also increased for the stimulation $\checkmark$ the digits of the left hand of musicians compared to the left hand of controls [ANOVA interaction of group and side of stimulation, $F(1,13)=5.54, P<0.05]$. The increase was larger for D5 $(t=5.4, P<$ 0.01) than for D1 $[t=2.0, P<0.1$; ANOVA interaction of group and digit, $F(1,13)=4.81, P<0.05](19)$.

There was a correlation between the age at which the string players began studying their instruments and the magnitude of the change in the dipole moment of D5 compared to that in controls $(r=0.79, P=$ $0.01)$ (Fig. 1B). The relation between the amount of practice and the cortical measures was not significant. The increase of the dipole moment of both D1 and D5 in the string players indicates that an extended cortical network responds to tactile stimulation. If the active area expands, the point location determined by an equivalent "nole model will shift inward. Given the herical geometry of the head, measurements of the D1 and D5 representations would approach each other if the center of activity remained unchanged. Conversely, an increase in area of representation would produce opposing effects on the measured cortical distance between D1 and D5, which could explain the absence of a significant group difference in the measured distance between cortical representations of D1 and D5 (Fig. 1C). Further experiments should attempt to model the size of the activated area to resolve this question.

The significant shift in medial direction of the cortical representation of the fingers of the left hand in string players and the increase in the corresponding dipole moments suggest that the cortical territory oc-

T. Elbert and B. Rockstron. Department of Psychology, University of Konstanz, D-78434 Konstanz, Germany. E-mail: Thomas .Elber@@Uni-Konstanz.de

C. Pantev and C. Wienbruch, Biomagnetism Center, University of Münster, D-48129 Münster, Germany.

E. Taub, Department of Psychology, University of Ala bama at Birmingham, Birmingham, AL 35294, USA. cupied by the representation of the digits increased in string players as compared with that in controls. Two alternate interpretations of our data should be taken into account. First, it could be argued that individuals with a genetically determined large represcntation of the left hand digits make superior string players and therefore are more likely to continue with musical training once they have begun. Howcver, in research with animals, use-dependent enlargements of portions of the somatosensory map in cortical area 3 b have been clearly demonstrated under conditions of increased use generally similar to those in this study $(12,13)$. In either case, the relatively larger representations of individually important digits could have the role of enhancing the particular needs of a string player in an adaptive manner.

A second altcrnative explanation of our results is that thcy are a consequence of a shift in cortical responsivity combined with an intensification of the response. However, we think a more plausible explanation is that the cortical territory of the left-hand digits has expanded. This is more plausible because (i) there is a correlation between amount of cortical reorganization and age [or stage of central nervous system (CNS) maturation] at which musical practice began and (ii) the equivalent current dipole (ECD) shift follows the one direction that is consistent with the expansion interpretation.

Related work (10) has shown that there is a strong correlation in humans with upper extremities amputated between extent of cortical reorganization and amount of phantom limb pain experienced. Although phantom limb pain is a maladaptive result of nervous system injury, our results demonstrate the functional relevance of cortical reorganization, similar to results reported in the context of the visual system (3) and auditory system $(12,20,21)$. One may speculate that one role of cortical reorganization might be to contribute to the functional recovery of organisms after CNS damagc, possibly in terms of recovery from CNS shock. The evolutionary advantage of this mechanism is brought into question, howcver, by the fact that the process of recovery is usually slow and thus would not permit an organism that was seriously impaired to survive long enough to engage in successful reproduction and transmission of this capacity (22).

However, in accord with the fesults of Merzenich and co-workers (7), continuous plastic reorganization of cortical space that permits rapid reallocation of available CNS circuitry would confer an obvious practical advantage. The possible contribution of cortical reorganization to rccovery of function after CNS injury might thus be an adventitious result that "piggy backed" onto a mechanism, which permitted the much more critically important plastic processes associated with learning, physical growth during maturation, and adjustment to current environmental demands. The tole of this mechanism in the recovery of function would become important only when protecting an individual with CNS damage would artificially prolong survival long enough for extensive cortical reorganization to work.

\section{REFERENCES AND NOTES}

\section{J. H. Kaas, Annu. Rev. Neurosci. 14, 137 (1991).}

2. A. Mogilner et al., Proc. Natl. Acad. Sci. U.S.A. 90 3593 (1993); M. M. Merzenich et al., Neuroscience 8, 33 (1983); S. A. Clark, T. Allard, W. M. Jenkins, M. M. Merzenich, Nature 332, 444 (1988); J. Kalaska and B. Pomeranz, J. Neurophysiol. 42, 618 (1979); D. D. Rasmusson, I. Comp. Neurol. 205, 313 (1982); A. M. Kellohan and G. S. Doetsch, Somatosens. Res. 2, $49(1984) ;$ J. T. Wall and C. G. Cusick, J. Neurosci. 4, 1499 (1984); J. T. Wall et al., ibid. 6, 218 (1986).

3. C. Darian-Smith and C. D. Gilbert, Nature 368,737 (1994)

4. C. D. Gilbert and T. N. Wiesel, ibid. 356, 150 (1992); F. Rösler, B. Röder, M. Heil, E. tienninghausen, Cognit. Brain Res. 1, 145 (1993)

5. G. H. Recanzone, C. E. Schreiner, M. M. Merzenich, J. Neurosci. 13, 87 (1993); D. Robertson and D. R. F. Irvine, J. Comp. Neurol. 282, 456 (1989); D. R. F. Irvine, R. Rajan, L. Z. Wize, P. Heil, Soc. Neurosci. Abstr. 17, 1485 (1991).

6. L. G. Cohen, S. Bandinelli, T. W. Frindley, M. Hallet, Brain 114, 615 (1991); J. N. Sanes, S. Suner, J. F Lando, J. P. Donaghue, Proc. Natt. Acad. Sci. U.S.A. 85, 2003 (1988)

7. M. M. Merzenich et al., J. Comp. Neurol. 224, 591 (1984)

8. T. P. Pons et al., Science 252, 1857 (1991).

9. T. Elbert et al., Neuroreport 5; 2593 (1994).

10. H. Flor et al. Nature 375, $482(1995)$

11. T. T. Yang et al., Neuroreport 5, 701 (1994)

12. G. H. Recanzone, M. M. Merzenich, W. M. Jenkins, A. Grajski, H. R. Dinse, J. Neurophysiol. 67, 1031 (1992).

13. W. M. Jenkins, M. M. Merzenich, M. T. Ochs, T. Allard, E. Guic-Robles, ibid. 63, 82 (1990).

14. A. Pascual-Leone and F. Torres, Brain 116, 39 (1993).

15. Controls were either students or university employ ees who worked in an academic environment and had no experience playing string instruments. They were selected to be comparable to the experimental group with respect to age, social status, and genider. After giving informed consent, experimental participants were briefly interviewed concerning their musicai activities and their history of practicing. Experimerital participants were also interviewed to elici information regarding their usual manual activities: None of the controls engaged in manual tasks to an unusual extent other than typing on computer keyboards. Each of them used both hands for word processing or other computer operations. Two of the musicians were found to have a tendency toward ambidexterity, but all other test participants wero right-handed (Edinburgh handedness questionnaire) [R. C. Oldfield, Neuropsychologia 9, 97 (1971)].

16. T. T. Yang, C. C. Gallen, B. J. Schwart, F. E. Blcom Proc. Natl. Acad. Sci. U.S.A. 90, 3089 (1993)

17. A BTi-Magnes system was used for MEG (magnetoencephalographic) recordings. The sensor array was positioned over one of the two hemispheres (C3 or C4) in a fixed irregular order. At each site, 1000 stimuli were delivered at an average rate of $0.5 \mathrm{~Hz}$ (the interval between stimulus onsets was $500 \pm 50$ ms). Stimulation site sequence varied according to a fixed irregular order across test participants. Within the range of 30 to $75 \mathrm{~ms}$, a first major peak was identified in each of the evoked wave forms. The mean latencies were $46.2 \pm 7.9 \mathrm{~ms}$ for left D1, and $48.1 \pm 7.2 \mathrm{~ms}$ for right $\mathrm{D} 1$; for left $\mathrm{D} 5$, the mean 
latency was $52.2 \pm 8.3 \mathrm{~ms}$, and for right D5 $52.8 \pm$ $7.5 \mathrm{~ms}$. The difference between musicians and controls for latency was not significant. For each evoked magnetic field, a single ECD model (best fitting local sphere) was fitted and the medians of the dipole moment and the dipole location were computed from a selection of points within a 20 -ms time seg ment (11 sampling points) around the maximal rms (root mean square across the 37 channels) within the range of 30 to $75 \mathrm{~ms}$. Points were selected if they met the following requirements: (i) rms indicating a signal-to-noise ratio > 3 ; (ii) a goodness of fit of the ECD model to the measured field $>0.95$; and (iii) a minimal confidence volume of the ECD location $<300 \mathrm{~mm}^{3}$

18. For all cortical measures, an ANOVA with the between-subject factor group (musicians versus controls) and the within-subject factors digit (D1 versus D5) and side of stimulation (left versus right) was computed first. ANOVAs for subsets of the data or $t$ tests were used to resolve interactions.

19. Given a constant direction of the equivalent current dipole, the dipole moment indicates the total strength of cortical polarization - that is, the number of neurons involved during a cortical response. If this number increases, the dipole moment also increas es. Any active focal area can be modeled by an equivalent current dipole. Each dendritic current flow contributes to this dipole moment according to the formula

dipole moment $=$ (conductivity)

$x$ (cross section of the dendrite)

$\times$ (potential difference along the dendrite)

S. J. Williamson and L. Kaufman [in Auditory Evoked Magnetic Fields and Electric Potentials, F. Grandori, M. Hoke, G. L. Romani, Eds. (Karger, Basel, 1990), pp. 1-39] assume the diameter of an apical dendrite to be $4 \mu \mathrm{m}$, the intracellular conductivity to be about
$0.25 \mathrm{~S} / \mathrm{m}$, and the potential difference to be about 10 $\mathrm{mV}$. With the use of these assumptions, about 30,000 dendrites would be necessary to produce a dipole moment of $10 \mathrm{nA} \cdot \mathrm{m}$. If conductivity and potential difference are not different in musicians and controls, the magnification of the dipole moment in response to finger stimulation of the left hand in musicians can be explained if approximately twice as many cells were activated in musicians than were activated in the controls.

20. G. H. Recanzone, M. M. Merzenich, J. Schreiner, J Nourophysiol. 67, 1071 (1992)

21. N. M. Weinberger et al, Concepts Neurosci. 1, 91 (1990).

22. We are indebted to $T$. Pons for this observation.

23. We appreciate the assistance of $\mathrm{S}$. Hampson, $\mathrm{B}$. Lütkenhöner, and $O$. Steinsträter. Supported by the Deutsche Forschungsgemeinschaft.

25 May 1995; accepted 13 September 1995 